\title{
Competencia digital docente para la reducción de la brecha digital: Estudio comparativo de España y Costa Rica
}

\section{Teacher's Digital Competence for Reducing Digital Divide: Comparative Study Between Spain and Costa Rica}

\author{
Ana Pérez-Escoda \\ Universidad Nebrija (España) \\ Ana Iglesias-Rodríguez \\ Universidad de Salamanca (España) \\ Lady Meléndez-Rodríguez \\ Viviana Berrocal-Carvajal \\ Universidad Estatal a Distancia (Costa Rica)
}

La inmersión de la sociedad en la era digital ha provocado transformaciones irrefrenables como la conectividad continua, el big data o la inteligencia artificial, que implican una mediatización tecnológica de todas las interacciones humanas y que requiere una formación en competencias específicas. El objetivo principal del estudio se centró en realizar un análisis comparativo internacional en dos países con contextos diferentes: España y Costa Rica, para demostrar que la globalización de las TIC y la conectividad han hecho que el profesorado sea piedra angular en el desarrollo de competencias digitales como garantía para la reducción de las brechas digitales por uso y no por acceso. Con una metodología cuantitativa
The immersion of society in the digital age has caused unstoppable transformations such as continuous connectivity, big data or artificial intelligence, which imply a technological mediation in all human interactions and which require training in specific skills. The main objective of the study focuses on conducting an international comparative analysis between two countries within different contexts, Spain and Costa Rica, to demonstrate that the globalization of ICT and connectivity have made teachers a cornerstone in the development of digital skills as they are a guarantee for the reduction of the digital gap in the use and not in the access. Using a quantitative methodology of descriptive-correlational character, 
de carácter descriptivo correlacional se analizaron cinco constructos de estudio en una muestra de 126 docentes procedentes de ambos países. Los resultados evidencian que pese a las diferencias políticas, sociales y educativas de cada país la percepción de los docentes no es tan diferente. La conclusión principal es que los docentes en ambos países se sienten seguros de sus capacidades digitales, están motivados, entienden que el sistema educativo no responde a las necesidades actuales y ven necesaria una inclusión curricular específica de la materia.

Palabras clave: competencia digital, brecha digital, profesorado, TIC, formación, educación. five study constructs were analyzed in a sample of 126 teachers from both countries. The results show that despite the political, social and educational differences in each country, the perception of teachers is not so different. The main conclusion is that teachers in both countries feel confident in their digital skills, are motivated, understand that the educational system does not respond to current needs, and they feel that a specific curricular inclusion of the subject is needed in formal education.

Key words: digital competence, digital divide, teachers, ICT, training, education.

\section{ESTADO DE LA CUESTIÓN}

Cien años fueron necesarios para que, desde que el telégrafo y el teléfono irrumpieran en la historia de la humanidad, mil millones de sitios físicos estuvieran comunicados. Sin embargo, tan solo han bastado treinta años para que 50.000 millones de dispositivos estén interconectados (Palfrey y Gasser, 2010). La inmersión tecnológica a escala global es, sin duda, una de las grandes evidencias de que la sociedad red es ya una realidad, por lo que si hace dos lustros la preocupación a escala global eran las brechas de acceso a tecnologías digitales, en la segunda década del siglo XXI la inquietud se centra en la brecha digital por uso eficiente de esa tecnología (Alva de la Rosa, 2015; Deursen, 2017; Deursen et al., 2019). El vertiginoso avance de la tecnología actual impone soluciones rápidas y adecuadas que doten a la población en general de respuestas formativas efectivas que le hagan estar preparada ante los retos de una nueva era marcada por la inteligencia artificial, el big data y el 5G (Aguaded, Marín-Gutiérrez y Caldeiro-Pedreira, 2018).

Tomando como punto de partida que la educación debe diseñarse para promover y fomentar las capacidades humanas en el desarrollo de una alfabetización digital y que debe mejorar las habilidades requeridas en la era digital, reformularse y repensarse desde una nueva perspectiva de alfabetización y competencias (Castañeda, Esteve y Adell, 2018). Así ha quedado ratificado una y otra vez en el marco internacional de reconocimiento de competencias necesarias para la 
formación continua de la ciudadanía (European Commission, 2018; ISTE, 2008;

OCDE, 2005; Pérez-Escoda y Fernández-Villacencio, 2016; Pettersson, 2018).

No obstante, la transformación de la educación que, como derecho universal y objetivo de desarrollo sostenible (UNESCO, 2019), está en manos de países y gobiernos, así como decisiones políticas, queda en último término y de modo global bajo la responsabilidad de los docentes que, como garantes de la educación, impulsan y desarrollan desde el aula las verdaderas innovaciones (Blau y Shamir-Inbal, 2017).

Así pues, el empoderamiento de la ciudadanía ante competencias emergentes y nuevas alfabetizaciones se encuentra, en parte, atesorado bajo el dominio de los profesores, quienes deben asumir tácita o implícitamente el reto de procurar un acceso universal a la tecnología y a las competencias que garantizan su dominio (Ilomäki et al., 2016; Cabero-Almenara y Martínez-Gimeno, 2019; UNESCO, 2018). Este reto comienza con el compromiso del profesorado y el esfuerzo constante de este colectivo por adecuarse a las necesidades educativas que el contexto digital impone, con una particularidad con respecto a la década anterior: las tecnologías se han hecho presentes de modo global y extensivo hasta el punto de convertirse en elementos fundamentales de uso en cualquier parte del mundo. Si centramos la atención en los dos contextos estudiados en este trabajo, encontramos que 9 de cada 10 latinoamericanos tienen teléfono móvil y en el 44\% de los casos se trata de un smartphone (Basco, 2017), por lo que la brecha digital de acceso está prácticamente superada. En el caso de España, se alcanzan cuotas de mercado para el smartphone del 92\% con 114 líneas de teléfono móvil por cada 100 habitantes, lo que supone un mercado saturado y una inexistencia en la brecha digital de acceso (Telefónica, 2018). Por tanto, las brechas de acceso están cada vez más superadas debido a la penetración de la tecnología móvil a escala global, lo que pone el foco de atención en otro problema - las brechas digitales de uso propiciadas por inercias de acceso a la tecnología que afectan a todos los nichos poblacionales (Holloway, Green y Livingstone, 2013; Masanet, Guerrero-Pico y Establés, 2019)— e impone tratar la situación desde una perspectiva educativa.

\section{COMPETENCIAS DigitAlES DEL PROFESORADO: UNA PREOCUPACIÓN} INTERNACIONAL

La inercia internacional hacia la definición y conceptualización de competencias que aúnen necesidades formativas y laborales y acorten brechas digitales constituye uno de los focos fundamentales en las agendas de políticas y centros educativos en todo el mundo (European Commission, 2018; INTEF, 2017; Programa Estado de la Nación, 2017). En el contexto de ambos países elegidos para la muestra objeto de estudio, España y Costa Rica, se encuentra una línea común en las políticas educativas desde comienzos de siglo, especialmente enfocadas en el compromiso de un desarrollo de las competencias digitales en el ámbito educativo y, más concretamente, en los docentes como premisa de partida y aspecto fundamental en el empoderamiento de la ciudadanía y garantía de desarrollo de una economía digital. 
En el contexto concreto de España se encuentra una preocupación desde principios de siglo por establecer marcos estratégicos y acciones en política educativa TIC que, si bien al principio, se caracterizaron por estar descentralizadas, fue a partir de 2008 con la aparición del Instituto Superior de Formación y Recursos en Red para el Profesorado (ISFTIC) que comenzaron una serie de planes centrados en el desarrollo de una todavía no reconocida competencia digital: Plan Info XXI, España.es, Plan Avanza 1, Escuela 2.0 y Plan Avanza 2. Será en este último plan, donde, al amparo del marco de referencia europeo, la Agenda Digital para España y, dentro de ella el Programa de Educación Digital, se contemplará, por primera vez, como medida fundamental en la transformación de la educación, el desarrollo de la competencia digital docente (2013-2015). Con el fin de concretar el desarrollo de estas medidas, el 4 de octubre de 2012 se decidió elaborar el Plan de Cultura Digital en la Escuela, espacio de colaboración y decisión conjunta entre el Ministerio de Educación, Ciencia y Deporte (MECD) y las Comunidades Autónomas. El proceso de reflexión se estructuró en cinco grupos de expertos vinculados a los cinco proyectos prioritarios del plan, uno de los cuales era la competencia digital docente. Desde este momento se trabajó continuamente en diferentes propuestas siendo la última versión el Marco Común de Competencia Digital Docente en octubre de 2017 (INTEF, 2017).

En el ámbito de Costa Rica, desde 2004 se creó la Red Latinoamericana de Portales Educativos (RELPE), como un acuerdo de cooperación regional de políticas de informática educativa, que comprometía a las autoridades educativas de 17 países latinoamericanos, incluido Costa Rica, a mejorar el uso de las TIC en educación, en tanto que no se creía que la dotación TIC en los centros educativos estuviera logrando los objetivos propuestos en términos didácticos ni de mejoramiento del rendimiento de los alumnos. Por otro lado, el Plan Nacional de Desarrollo de las Telecomunicaciones, del Ministerio de Ciencia, Tecnología y Telecomunicaciones (MICIT), planteó en 2015 políticas nacionales centradas en la inclusión, el desarrollo de la economía digital y el gobierno electrónico y transparente, donde se destacaba la atención a grupos en situación de vulnerabilidad mediante el desarrollo de acciones focalizadas que les permitieran mejorar sus condiciones de acceso a las telecomunicaciones/TIC (Programa Estado de la Nación, 2017). Estas acciones traslucían un reconocimiento implícito por parte del MICIT del impacto positivo que las TIC podían tener en el conocimiento y condición socioeconómica de las personas, sobre todo de los grupos en condición de vulnerabilidad, siempre y cuando pudieran desarrollar adecuadas competencias para su uso. Costa Rica ha sido y es un país preocupado por el desarrollo de competencias digitales como atenuante de las brechas digitales generadas por la tecnología (en un primer momento de acceso y actualmente de uso eficiente); así, en las Metas educativas 2021 (OEI, 2009) se destacaba el alto nivel que mostraban Chile, Uruguay y Costa Rica en cuanto a la disponibilidad de computadores, señalando que la principal tarea del sector educativo era construir una relación efectiva entre estudiantes, profesores, contenidos y evaluación de los aprendizajes en un nuevo escenario planteado desde las TIC para un mejor rendimiento (PROSIC, 2019).

Las diferentes acciones nacionales al respecto han dado lugar a propuestas varias que describen competencias relacionadas con la práctica digital de una 
alfabetización diferente y más adecuada al contexto actual, una alfabetización digital basada en las competencias necesarias en el siglo XXI (Castañeda, Esteve y Adell, 2018; Pérez-Escoda, Aguaded y Rodríguez-Conde, 2016; Pérez-Escoda, García-Ruiz y Aguaded, 2019). Para la realización de este estudio se han tomado como referencia diferentes marcos competenciales por el carácter internacional del trabajo, pero todos ellos relacionados con el ámbito educativo (Partnership for 21st Century Skills, 2009; ISTE Standards y DigCompEdu (Redecker y Punie, 2017) y que son los que se muestran en el gráfico 1.

\section{Gráfico 1. Marcos de referencia internacional para la competencia digital}

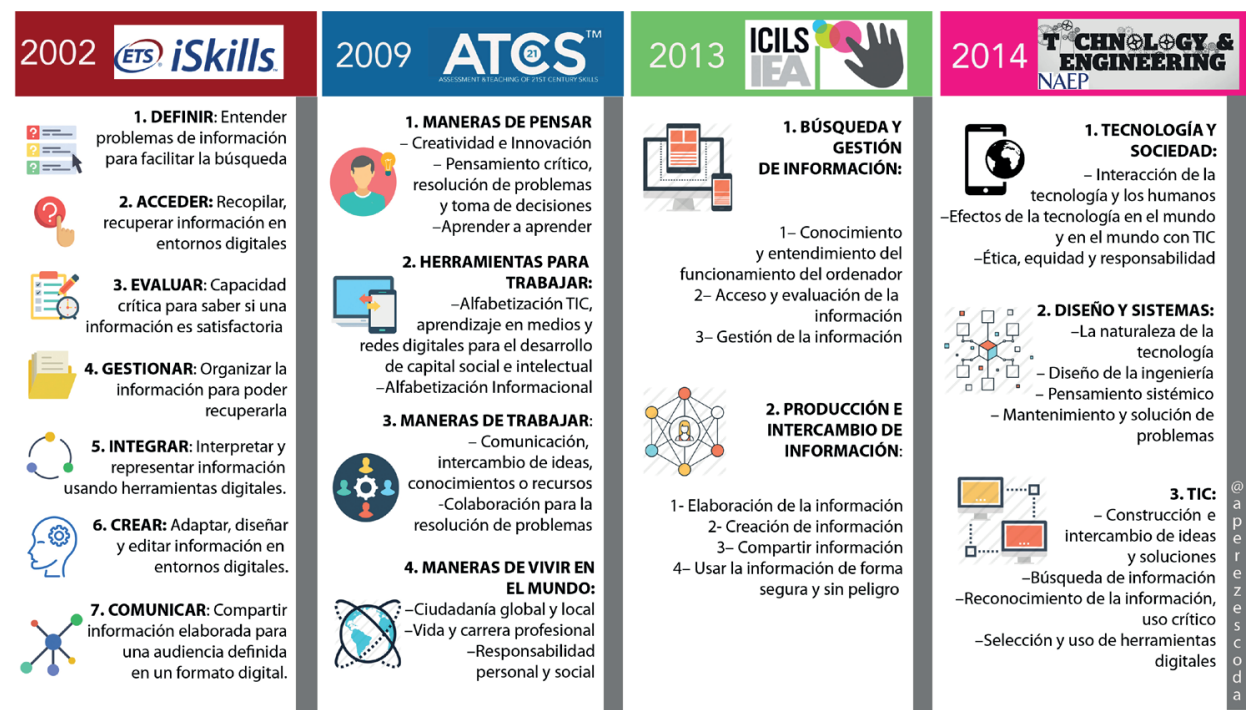

Fuente: elaboración propia.

El estudio de estas propuestas sirvió de apoyo en la definición de cinco constructos de investigación con diferentes ítems que dieran sentido a la investigación planteada para ambos contextos estudiados: España y Costa Rica. Puesto que el tema tratado es de alcance internacional, el objetivo principal de estudio se centró en realizar un análisis comparativo internacional que recogiera datos empíricos de la situación del profesorado de educación primaria en dos países con contextos muy diferentes para demostrar que la globalización de las TIC, el alcance de la tecnología y la conectividad han hecho que la formación del profesorado sea piedra angular en el desarrollo de competencias digitales como garantía para la reducción de las brechas digitales por uso y no por acceso o formación. El dominio de las competencias digitales por parte del profesorado, así como su grado de concienciación al respecto, supondrá garantía de empoderamiento digital de los pueblos frente a la desigualdad, pues las nuevas generaciones se encontrarán con iguales posibilidades de participación y acceso si acceden a una formación adecuada en el ámbito digital (Ilomäki et al., 2016; Krumsvic, 2014; Livingstone et al., 2017; Van Deursen y Van Dijk, 2016). 
El estudio de las competencias digitales ha sido abordado en la última década desde la perspectiva de los docentes en la educación superior (MuñozCarril, González-Sanmamed y Fuentes-Abeledo, 2011; Carrera y Coiduras, 2012; Esteve y Gisbert, 2013), en la formación inicial del profesorado no universitario (Girón-Escudero; Cózar-Gutiérrez y González-Calero, 2019; Güneş y Bahçivan, 2018) y en la formación del profesorado universitario (Blau y Shamir-Inbal, 2017; Instefjord y Munthe, 2016; Cabero-Almenara y MartínezGimeno, 2019) como elemento consustancial a la formación en la sociedad de la información (García-Ruiz y Pérez-Escoda, 2019), por lo que también resulta necesario ampliar el campo de estudio a profesorado de educación obligatoria en activo.

\section{MATERIAL Y MÉTODOS}

El estudio que se presenta de corte cuantitativo de tipo descriptivo-correlacional se basó en la adaptación de una encuesta existente (Pérez-Escoda, Aguaded y Rodríguez-Conde, 2016) donde las variables de estudio trabajadas resultaron idóneas para el estudio comparativo España-Costa Rica. La encuesta utilizada resultaba especialmente conveniente por estar basada en las evaluaciones más relevantes sobre competencias digitales percibidas como son iSKILLS Assessment (Educational Testing Service, 2002); ATCS21, Assessment and Teaching of 21st Century Skills (Binkley et al., 2012); ICILS, InternationalComputer and Information Literacy Study (Fraillon, Schulz y Ainley, 2013); TEL, Technology and Engineering Literacy (National Assessment Governing Board, 2014). El instrumento para la recogida de datos se adaptó teniendo en cuenta la última modificación del marco europeo para el desarrollo y definición de las competencias digitales en educación -DigCompEdu (Redecker y Punie, 2017) - específicas para los docentes. Además, el número de ítems estudiados, un total de 62 , se asemejó a otros instrumentos validados para estudios similares como el de Tourón et al. (2018). De este modo, el cuestionario se diseñó y organizó en cinco clusters, que conformaron los constructos de estudio como se muestra en el gráfico 2 .

\section{Gráfico 2. 'Clusters' de estudio}

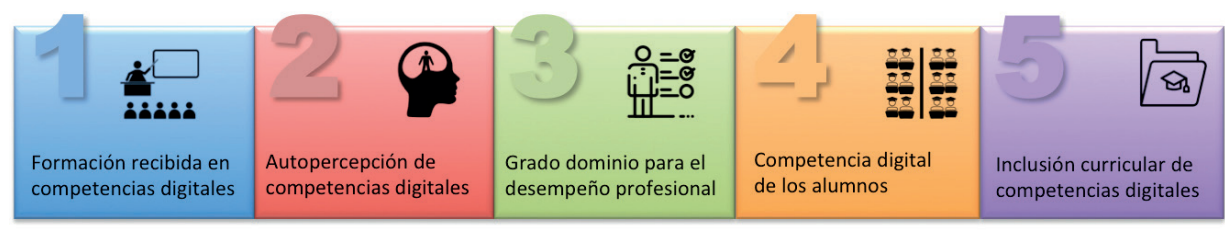

Fuente: elaboración propia.

Los ítems incluidos en el cuestionario final abordaron los siguientes aspectos relevantes en este tipo de estudios: 1) formación recibida en competencias digi- 
tales; 2) autopercepción de las competencias digitales propias; 3) grado de dominio de estas competencias para el desempeño profesional; 4) grado de competencia digital de los alumnos y, 5) inclusión curricular de las competencias digitales.

Es importante destacar que, para la medición de variables de estudio de esta naturaleza que llevan asociada una complejidad añadida por el cambio continuo que entraña la naturaleza misma de las competencias digitales en el contexto de la sociedad de la información, resulta fundamental garantizar la validez y fiabilidad del instrumento elegido para la recogida de datos. Para garantizar el grado de fiabilidad se aplicó el índice de consistencia interna Alfa de Cronbach de manera separada para los cinco constructos estudiados, con un total de 62 ítems, con respuesta tipo Likert en una escala de cuatro posibles respuestas, donde 0 era igual a nada y 4 equivalía a mucho, y que constituyeron el cuestionario final, de lo que se obtuvo en el caso de España un valor para el coeficiente de Cronbach de 0,85 y en el caso de Costa Rica un 0,80 , siendo en ambos casos $>0.70$, valores que se consideran suficientes para garantizar la fiabilidad del instrumento utilizado para los dos países (Bisquerra, 2004).

\section{MUESTRA Y CARACTERIZACIÓN DE LA MUESTRA}

La muestra estuvo compuesta por 126 docentes, el muestreo se realizó por conveniencia en ambos países, en los que se contó con 63 docentes españoles y 63 docentes costarricenses, todos ellos docentes en escuelas de educación primaria en activo. La descripción de la muestra según diferentes parámetros nos indica que tenemos muestras parecidas. El análisis de la muestra se realizó en función de las variables: género, edad, años de experiencia, formación y número de centros participantes. En cuanto al género, 3 de cada 4 profesores son mujeres en España, mientras que ascienden a 9 de cada 10 en Costa Rica (p-valor $\chi^{2}$ 0.009). Respecto a la edad, el año de nacimiento es, en promedio, de 1972 en España al igual que en Costa Rica (p-valor<0.05). Es decir, la edad media del profesor es de 47 años en ambos países. En cuanto a la variable experiencia, los años de experiencia son, de promedio, 16 en España y 17 en Costa Rica, no habiendo diferencias significativas entre ambas cifras. En España, el 94\% de los profesores son diplomados (magisterio principalmente), mientras que el 62\% de los profesores de Costa Rica no tienen diplomatura (p-valor $\chi^{2}$ 0.000). En España, el 25\% de los profesores son licenciados y en Costa Rica lo son el 89\% (sobre todo en I y II ciclo) (p-valor $\left.\chi^{2} 0.000\right)$. En España, el 25\% de los profesores tiene un grado, mientras que en Costa Rica este porcentaje aumenta hasta el 57\% (sobre todo en I y II ciclo) (p-valor $\chi^{2} 0.000$ ). El porcentaje de profesores con máster en España es del 11\% y en Costa Rica del $20 \%$, cifras estadísticamente iguales (p-valor $\chi^{2} 0.159$ ). El porcentaje de profesores con doctorado en España es del 5\% y en Costa Rica, del 2\%, cifras estadísticamente iguales ( $p$-valor $\chi^{2}$ 0.402). Si tenemos en cuenta la localización, en España se recogieron datos de 9 centros educativos distintos, mientras que en Costa Rica fueron 10 los centros de procedencia de los docentes encuestados. 


\section{ANÁLISIS Y RESULTADOS}

Presentamos ahora los resultados obtenidos a partir del análisis estadístico con el software SPSS en cuanto a los descriptivos estadísticos básicos relativos a los cinco constructos de estudio con un total de 62 ítems respondidos en ambos países para realizar el análisis comparativo entre ambos y analizar las diferencias estadísticamente significativas. Se presenta el análisis y los resultados por clusters.

\section{FORMACIÓN RECIBIDA EN COMPETENCIAS DIGITALES}

En España, el 97\% de los profesores ha recibido formación TIC, mientras que en Costa Rica solo el 44\% (p-valor $\chi^{2}$ 0.000). Tanto en España como en Costa Rica, la mayoría de profesores no son coordinadores TIC. En España, el 73\% de los profesores ha participado en algún proyecto de innovación TIC, mientras que en Costa Rica solo el 15\% (p-valor $\chi^{2}$ 0.000). Además, respecto a la formación recibida se distinguió entre formación permanente y formación por iniciativa propia, siendo los resultados como se muestran a continuación en el gráfico 3.

\section{Gráfico 3. Formación permanente recibida por país}

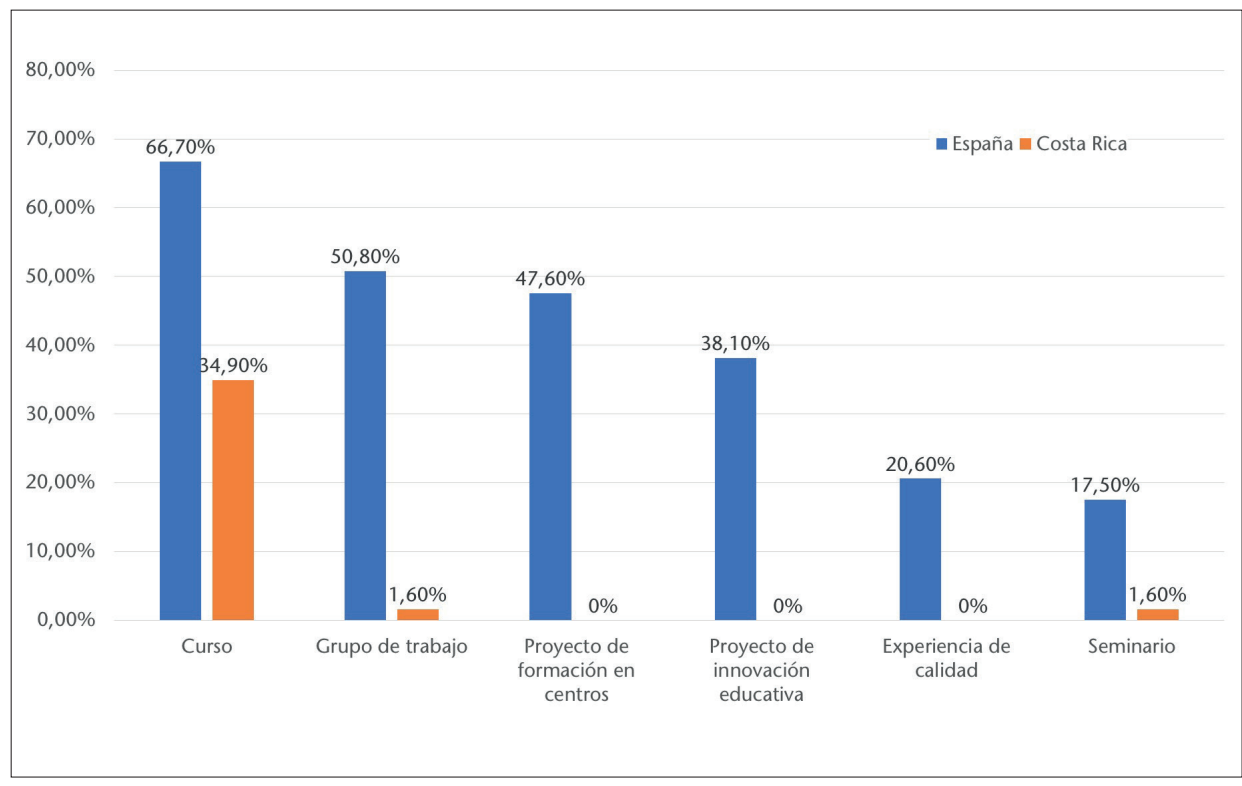

Fuente: elaboración propia.

El 67\% de los profesores españoles ha realizado algún curso de formación permanente, el 51\% ha recibido algún grupo de trabajo, el 48\% ha participado en algún proyecto de formación en centros. En Costa Rica, solo el 35\% de los profesores reconoce haber realizado algún tipo de formación permanente y ha sido un curso (hay un elevado porcentaje de "No responde" en Costa Rica, en torno al 25-30\%). 
Gráfico 4. Formación por iniciativa propia por país

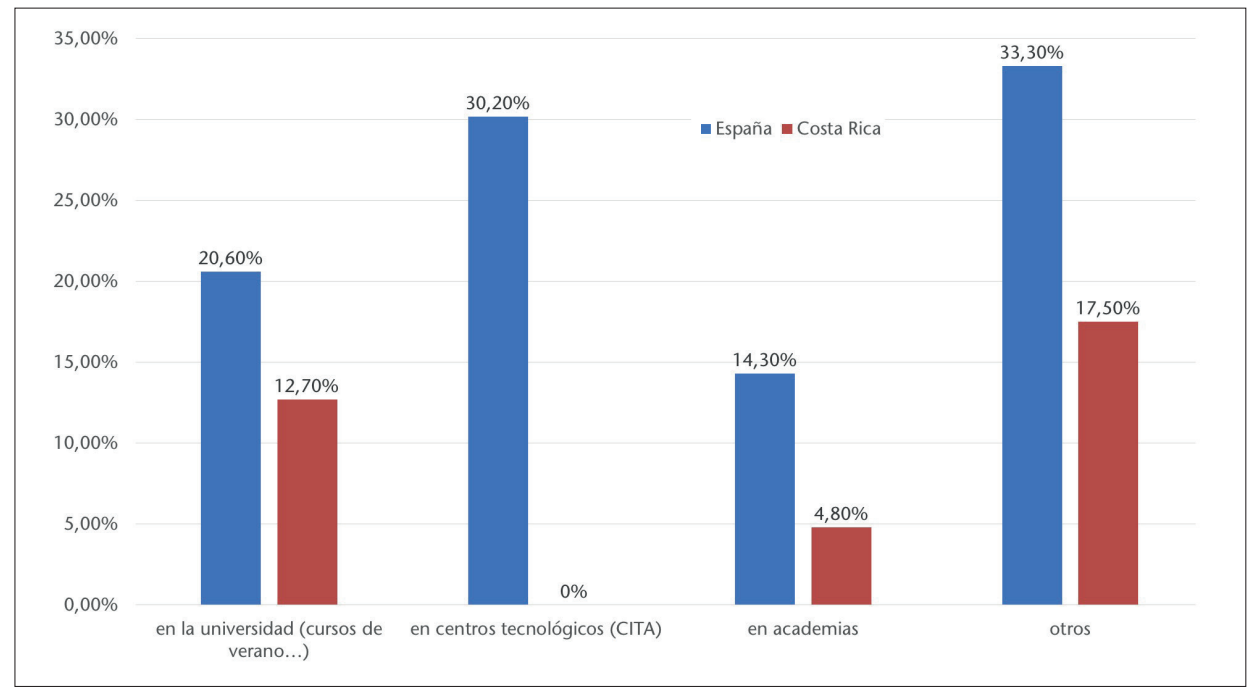

Fuente: elaboración propia.

Entre un 15 y un 35\% de los profesores se ha formado por iniciativa propia en España, siendo los centros tecnológicos y otros métodos los más frecuentes. En Costa Rica, el porcentaje de profesores con formación por iniciativa propia no alcanza el 20\%, siendo otros métodos el más frecuente (p-valor $\chi^{2} 0.000$ ).

\section{AUTOPERCEPCIÓN DE COMPETENCIAS DIGITALES}

En el análisis comparativo de este constructo encontramos resultados interesantes estudiados por áreas de la competencia digital divididas en información, comunicación, creación de contenido, seguridad y resolución de problemas (INTEF, 2017; Redecker y Punie, 2017), como pueden observarse en la tabla 1.

Tabla 1. Resultados de las competencias digitales autopercibidas por áreas

\begin{tabular}{|c|c|c|c|c|c|c|}
\hline \multirow{2}{*}{\multicolumn{2}{|c|}{ Competencias digitales autopercibidas }} & \multicolumn{2}{|l|}{ Media } & \multicolumn{2}{|c|}{ Desv. Tip. } & \multirow{2}{*}{$\begin{array}{l}\text { p-valor } \\
\chi^{2}\end{array}$} \\
\hline & & \multirow{2}{*}{$\begin{array}{l}\text { España } \\
3,16\end{array}$} & \multirow{2}{*}{$\begin{array}{l}\text { Costa } \\
\text { Rica } \\
3,13\end{array}$} & \multirow{2}{*}{$\begin{array}{l}\text { España } \\
0,72\end{array}$} & \multirow{2}{*}{$\begin{array}{l}\text { Costa } \\
\text { Rica } \\
1\end{array}$} & \\
\hline \multirow{3}{*}{ 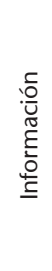 } & $\begin{array}{l}\text { Identificas y seleccionas información digital } \\
\text { (buscadores, metabuscadores, BBDD) }\end{array}$ & & & & & 0.187 \\
\hline & $\begin{array}{l}\text { Organizas y analizas la información digital para } \\
\text { evaluar su finalidad y relevancia }\end{array}$ & 1,97 & 1,71 & 1,09 & 1,54 & 0.001 \\
\hline & $\begin{array}{l}\text { Almacenas información digital (Evernote, } \\
\text { DIIGO...) }\end{array}$ & 3,13 & 3,29 & 0,89 & 1,01 & 0.128 \\
\hline
\end{tabular}




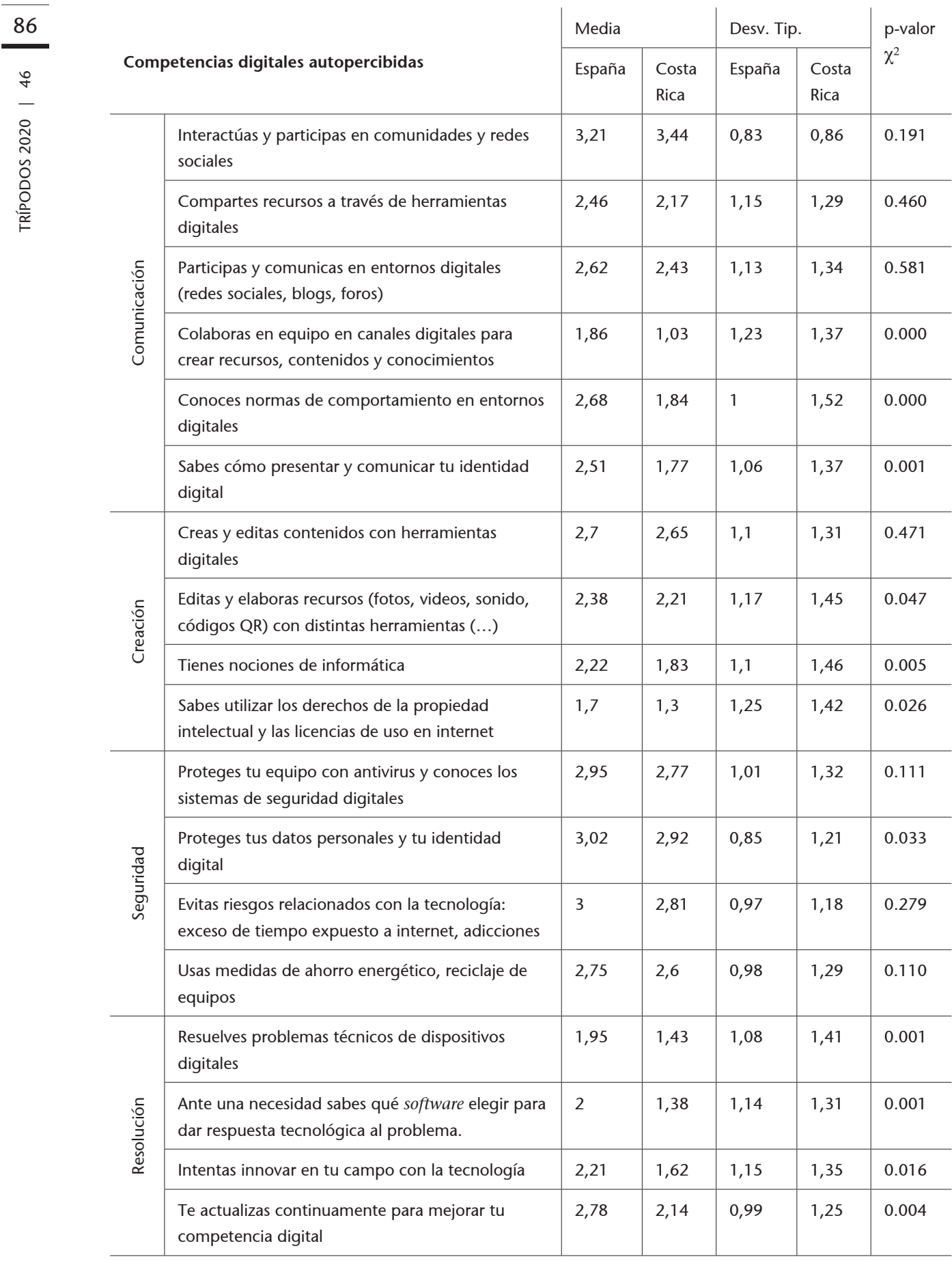


En el área de la información, los resultados en los valores de p>0.05 no muestran diferencias significativas entre ambos países en las competencias relativas a identificar, seleccionar y almacenar información digital, sin embargo, sí hay diferencia significativa en la organización y análisis de la información digital. En el área de la comunicación se encuentran diferencias significativas $(\mathrm{p}<0.05)$ en las competencias de trabajo colaborativo en red, normas de comportamiento en entornos digitales y configuración de la identidad digital, donde el grado medio de autopercepción de las competencias es bastante mayor en España. Respecto a la creación de contenido, aunque existen diferencias significativas en las nociones informáticas y los usos de los derechos de propiedad intelectual ( $\mathrm{p}<0.05$ ), destaca que en ambos países existe un grado muy bajo de uso de los derechos de propiedad intelectual. En el área de la seguridad, el grado de protección de los datos personales e identidad digital es menor en Costa Rica que en España p-valor $\chi^{2}$ 0.037, mientras que para el resto de competencias de esta área no hay diferencias significativas entre ambos países ( $p>0.05)$. Por último, en el área de resolución de problemas, el profesorado español tiene un mayor grado resolutivo, encontrándose diferencias significativas en las cuatro competencias analizadas $(\mathrm{p}<0.05)$ con valores de $\mathrm{p}=0.001,0.001,0.016$ y 0.004 , respectivamente.

\section{GRADO DE DOMINIO PARA EL DESEMPEÑO PROFESIONAL}

En cuanto al grado de dominio de la competencia digital para el desempeño profesional, cluster, que pretende ahondar en el nivel de la formación inicial del profesorado, aunque se encuentran diferencias significativas estadísticamente entre ambos países $(\mathrm{p}<0.05)$, la curva de resultados presenta prácticamente la misma forma. Se estudian ocho ítems a partir del enunciado: "Tu formación en competencia digital te hace sentir que..." 
Gráfico 5. Grado de dominio para el desempeño profesional con el estudio de las medias

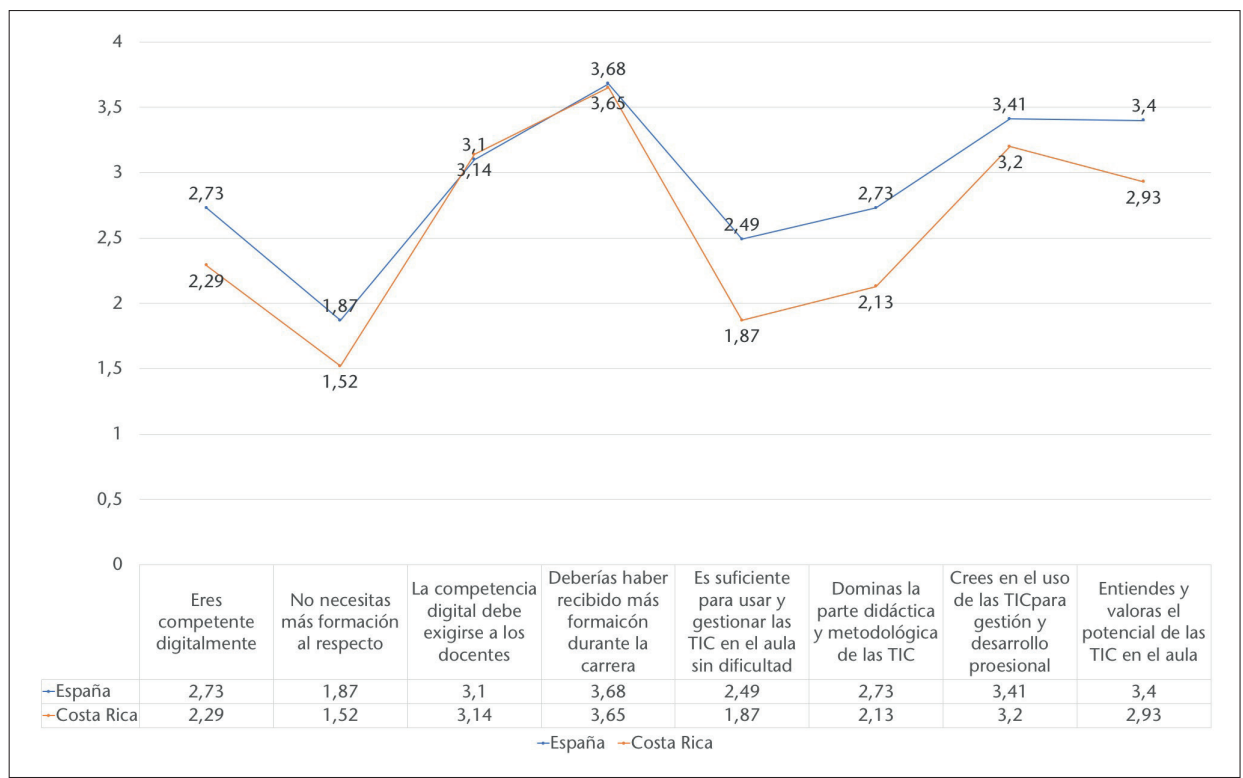

Fuente: elaboración propia.

\section{Competencias digitales de los Alumnos Al ACABAR los estudios OBLIGATORIOS}

Existen diferencias significativas en las valoraciones de todos los ítems entre países excepto en el ítem "han aprendido a proteger sus datos" como se aprecia en la tabla 2. Los profesores españoles creen, principalmente, que los alumnos de Educación Primaria han conocido normas básicas de comportamiento cuando navegan, gestión de la información en internet y pautas de comunicación digital.

Tabla 2. Percepción del profesorado sobre las competencias digitales de su alumnado

Con lo que aprenden los alumnos actualmente...

\begin{tabular}{|c|c|c|c|}
\hline & \multicolumn{3}{|l|}{ PAÍs } \\
\hline & Total & España & $\begin{array}{l}\text { Costa } \\
\text { Rica }\end{array}$ \\
\hline $\mathrm{N}$ válido & 124 & 63 & 61 \\
\hline Media & 2,32 & 2,44 & 2,20 \\
\hline Desviación estándar &, 94 & ,71 & 1,12 \\
\hline
\end{tabular}


Con lo que aprenden los alumnos actualmente...

\begin{tabular}{|c|c|c|c|}
\hline & \multicolumn{3}{|l|}{ PAÍS } \\
\hline & Total & España & $\begin{array}{l}\text { Costa } \\
\text { Rica }\end{array}$ \\
\hline $\mathrm{N}$ válido & 124 & 63 & 61 \\
\hline Media & 2,13 & 2,29 & 1,97 \\
\hline Desviación estándar & 1,01 & 79 & 1,18 \\
\hline
\end{tabular}

...conocen y usan herramientas para la creación de contenido textual

...han aprendido pautas de comunicación digital: chats, foros, redes, etc.

\begin{tabular}{|c|c|c|c|c|}
\hline & Desviación estándar & 1,01 & 79 & 1,18 \\
\hline \multirow{3}{*}{$\begin{array}{l}\text {...conocen y usan herramientas para la creación de } \\
\text { contenido textual }\end{array}$} & $\mathrm{N}$ válido & 124 & 63 & 61 \\
\hline & Media & 2,05 & 2,11 & 1,98 \\
\hline & Desviación estándar & ,99 & ,76 & 1,19 \\
\hline \multirow{3}{*}{$\begin{array}{l}\text {...conocen y usan herramientas para la creación de } \\
\text { contenido audiovisual }\end{array}$} & N válido & 125 & 63 & 62 \\
\hline & Media & 1,96 & 2,14 & 1,77 \\
\hline & Desviación estándar & 1,03 &, 80 & 1,19 \\
\hline \multirow{3}{*}{....han aprendido a gestionar su identidad digital } & N válido & 126 & 63 & 63 \\
\hline & Media & 1,67 & 1,86 & 1,48 \\
\hline & Desviación estándar & 1,14 & 1,00 & 1,24 \\
\hline \multirow{3}{*}{...han aprendido cómo proteger sus datos } & $\mathrm{N}$ válido & 125 & 63 & 62 \\
\hline & Media & 1,60 & 1,79 & 1,40 \\
\hline & Desviación estándar & 1,19 & 1,11 & 1,25 \\
\hline \multirow{3}{*}{$\begin{array}{l}\text {...han conocido normas básicas de comportamiento } \\
\text { cuando navegan }\end{array}$} & N válido & 126 & 63 & 63 \\
\hline & Media & 2,19 & 2,75 & 1,63 \\
\hline & Desviación estándar & 3,09 & 4,13 & 1,26 \\
\hline \multirow{3}{*}{$\begin{array}{l}\text {...han aprendido resolución básica de problemas: } \\
\text { distinguir información inapropiada, invitación de } \\
\text { extraños, soluciones informáticas básicas, etc. }\end{array}$} & N válido & 125 & 63 & 62 \\
\hline & Media & 1,93 & 2,14 & 1,71 \\
\hline & Desviación estándar & 1,17 & ,93 & 1,35 \\
\hline
\end{tabular}

Fuente: elaboración propia.

Los $\mathrm{p}$-valores de $\mathrm{p}<0.05$ indican que los profesores españoles piensan en mayor medida que los de Costa Rica que los alumnos conocen y usan herramientas para la creación de contenido audiovisual, han conocido normas básicas de comportamiento cuando navegan y han aprendido resolución básica de problemas: distinguir información inapropiada, invitación de extraños, soluciones informáticas básicas, etc.

\section{INCLUSIÓN CURRICULAR DE LAS COMPETENCIAS DIGITALES}

Con un total de diez ítems, este último constructo estudiado nos da una visión más concreta del sentimiento de los docentes objeto de estudio. 
Tabla 3. Percepción del profesorado sobre la inclusión curricular de las competencias digitales

\begin{tabular}{|c|c|c|c|c|c|c|c|}
\hline \multirow[t]{2}{*}{$\begin{array}{l}\text { Siendo consciente de lo que supone } \\
\text { la competencia digital crees que... }\end{array}$} & \multicolumn{2}{|l|}{ Media } & \multirow[t]{2}{*}{$\begin{array}{l}\text { p-valor } \\
\mathrm{Chi}^{2}\end{array}$} & \multicolumn{2}{|c|}{$\begin{array}{l}\text { En desacuerdo } \\
(\%)\end{array}$} & \multicolumn{2}{|c|}{$\begin{array}{l}\text { Bastante y muy } \\
\text { de acuerdo (\%) }\end{array}$} \\
\hline & España & $\begin{array}{l}\text { Costa } \\
\text { Rica }\end{array}$ & & $\begin{array}{l}\text { España } \\
N=63\end{array}$ & $\begin{array}{l}\text { Costa } \\
\text { Rica } \\
\mathrm{N}=63\end{array}$ & $\begin{array}{l}\text { España } \\
\mathrm{N}=63\end{array}$ & $\begin{array}{l}\text { Costa } \\
\text { Rica } \\
\mathrm{N}=63\end{array}$ \\
\hline $\begin{array}{l}\text { es suficiente con su integración } \\
\text { transversal en el currículo }\end{array}$ & 1,70 & 1,89 & 0.000 & 50,8 & 47,6 & 30 & 41,3 \\
\hline $\begin{array}{l}\text { debería desarrollarse más en el } \\
\text { currículo }\end{array}$ & 3,17 & 3,82 & 0.000 & 6,3 & 1,6 & 84,1 & 95,2 \\
\hline $\begin{array}{l}\text { es una materia necesaria en el } \\
\text { currículo del siglo XXI }\end{array}$ & 3,54 & 3,76 & 0.047 & 1,6 & 1,6 & 90,5 & 95,6 \\
\hline $\begin{array}{l}\text { es necesaria una revisión general del } \\
\text { currículo de primaria, pues los niños } \\
\text { en el siglo XXI necesitan otro tipo } \\
\text { de educación }\end{array}$ & 3,35 & 3,70 & 0.023 & 4,8 & 1,6 & 88,9 & 92,1 \\
\hline $\begin{array}{l}\text { la alfabetización digital debe } \\
\text { desarrollarse formalmente para } \\
\text { educar a ciudadanos competentes }\end{array}$ & 3,43 & 3,60 & 0.016 & 1,6 & 6,4 & 90,5 & 92,1 \\
\hline $\begin{array}{l}\text { dejar en manos de cada docente } \\
\text { la educación de la alfabetización } \\
\text { digital favorece la desigualdad } \\
\text { educativa }\end{array}$ & 2,92 & 2,71 & 0.016 & 12,7 & 22,2 & 76,2 & 60,3 \\
\hline $\begin{array}{l}\text { es conveniente la integración de la } \\
\text { alfabetización digital en el currículo } \\
\text { a partir de unos contenidos } \\
\text { previamente consensuados como } \\
\text { en el caso de otras materias: } \\
\text { matemáticas, lengua, etc. }\end{array}$ & 3,33 & 3,63 & 0.146 & 4,8 & 3,2 & 85,7 & 94,2 \\
\hline $\begin{array}{l}\text { un desarrollo formal ayudaría } \\
\text { y orientaría al profesorado y } \\
\text { fomentaría una educación al } \\
\text { respecto igualitaria }\end{array}$ & 3,29 & 3,70 & 0.013 & 12,6 & 3,2 & 87,3 & 93,6 \\
\hline $\begin{array}{l}\text { una metodología concreta y una } \\
\text { guía didáctica ayudaría a trabajar } \\
\text { todas las competencias digitales }\end{array}$ & 3,41 & 3,60 & 0.002 & 1,6 & 6,4 & 88,9 & 84,1 \\
\hline
\end{tabular}

Fuente: elaboración propia.

Los resultados traslucen que, en general, los profesores costarricenses están más de acuerdo en potenciar la competencia digital en el aula, sobre todo en aspectos como el desarrollo en el currículum, la revisión de dicho currículum, la alfabetización digital y un desarrollo formal de la materia. El último ítem de este bloque 
interrogó a los docentes en caso de que lo consideraran oportuno, en qué curso de primaria introducirían el aprendizaje de una alfabetización digital formal. En España, el 71,3\% del profesorado consultado introduciría este aprendizaje de modo explícito y específico en la primera etapa de primaria, y de ese porcentaje el 46\% lo haría en primero de primaria, es decir, a partir de los seis años. En Costa Rica, el parecer de los docentes es algo menor, el 68\% lo incluiría en este período y de este el 5,8\% lo haría en primero de primaria.

\section{CONCLUSIONES Y DISCUSIÓN}

Este artículo aporta nuevas evidencias, en la línea de estudios como los de Cabero-Almenara y Martínez-Gimeno (2019), Castañeda, Esteve y Adell (2018), PérezEscoda, Aguaded y Rodríguez-Conde (2016), Valdivieso y Gonzáles (2016), que proporcionan un conocimiento más profundo de la importancia que las competencias digitales del profesorado tienen para evitar y subsanar las brechas digitales de uso eficiente de las tecnologías.

El estudio de los cinco clusters analizados nos hace concluir que, en el primer constructo "formación recibida en competencias digitales", la diferencia entre el profesorado de ambos países resulta muy grande. En España, la preparación formal de los docentes es más del doble, por lo que se concluye que el profesorado español está mucho más preparado. Este dato debería corresponder a una autopercepción, por parte de los profesores españoles, muy alta, en la línea de trabajos como Güneş y Bahçivan (2018), que concluyen que a mayor formación mayor autopercepción, sin embargo, los datos recogidos en el segundo cluster no coincidieron plenamente con esta premisa.

El análisis de la "autopercepción de las competencias digitales" indica que el profesorado español posee un mayor grado en su capacidad de organización y análisis de la información digital, en las competencias colaborativas de trabajo en red y la creación de identidades digitales, así como la creación de contenidos digitales, resultados que refuerzan trabajos como el de Girón-Escudero; CózarGutiérrez y González-Calero (2019). Sin embargo, encontramos áreas de estudio como el uso de los derechos de autor o la resolución de problemas que se igualan a pesar de las desigualdades en la formación recibida.

Pese a los resultados en los dos primeros constructos analizados, el grado de dominio de estas competencias para el desempeño profesional resulta muy similar en ambos países, por lo que la conclusión principal es que, a pesar de las diferencias formativas y competenciales, los docentes buscan la manera de suplir sus carencias de cara a su desempeño profesional. El grado de competencia digital percibida de los alumnos por parte del profesorado es más alta en España que en Costa Rica, lo que explica tal vez los resultados del último constructo estudiado en el que los docentes en España expresan una mayor conciencia de la inclusión de estas competencias desde el inicio de la educación primaria.

Tal como han evidenciado estudios previos en el ámbito académico como los de From (2017) o Livingstone et al. (2017), el desarrollo de las competencias digitales, así como el nivel competencial del profesorado y su motivación hacia 
las TIC, son la clave para aprovechar en el aula un conocimiento espontáneo que se genera de modo innegable debido a la masiva exposición de los más jóvenes a la tecnología, tal y como se muestra en estudios nacionales, en España (AIMC, 2019) y en Costa Rica (PROSIC, 2019), como internacionales (Hootsuite, 2019). La visión comparativa del trabajo realizado permite una doble lectura: la primera, basada en el hecho de que, como traslucen los indicadores de la economía digital marcados por la OCDE (INEE, 2018) y alineados con los de la UIT (2018), las habilidades digitales son la piedra angular para el progreso de las sociedades basado en la formación y destrezas de la población; y la segunda, que las competencias digitales son la garantía de un acceso universal eficiente a dicha tecnología, cuyo aprovechamiento depende tanto del acceso como del uso inteligente.

Pese a las limitaciones del trabajo presentado, cuya metodología de muestreo es no probabilístico y, por tanto, no representativo del universo, supone un aporte empírico que evidencia que países con muy diferentes realidades en políticas e iniciativas tienen percepciones muy parecidas respecto al tema planteado y se sienten proactivos ante la problemática y el reto. El estudio supone un aporte más en la línea de investigaciones recientes (Blau y Shamir-Inbal, 2017; Deursen et al., 2019; Girón-Escudero; Cózar-Gutiérrez y González-Calero, 2019; Masanet, Guerrero-Pico y Establés, 2019) que dejan evidente el hecho de que la formación de las competencias digitales garantiza el acceso universal en igualdad de condiciones a las posibilidades que el siglo XXI nos brinda, y solo la concienciación a escala global, a gran escala y en todos los países conducirá a una verdadera disminución de las brechas digitales existentes.

Ana Pérez-Escoda (aperezes@nebrija.es). Doctora en Educación especializada en TIC y procesos comunicativos y formativos mediados por TIC. Profesora investigadora en la Facultad de Comunicación y Artes de la Universidad Nebrija, donde imparte docencia sobre comunicación digital, metodologías con TIC y procesos educativos mediados con TIC. Profesora colaboradora en el Programa de Doctorado Interuniversitario de Comunicación de la Universidad de Huelva. Ha sido coordinadora de Escuela de Doctorado y coordinadora de Formación e Innovación Docente (20152019) y editora asociada de la revista Comunicar. Miembro del Grupo de investigación en transformación digital Nebrija_INNOMEDIA. Es investigadora activa en el campo de la alfabetización mediática y digital, competencias digitales y transformación digital en el ámbito comunicativo y educativo.
Ana Iglesias-Rodríguez (anaiglesias@usal. es). Doctora en Pedagogía (Universidad de Salamanca, 2004). Profesora titular de Universidad. Actualmente trabaja en la Escuela Universitaria de Educación y Turismo de Ávila (Universidad de Salamanca). Como miembro del grupo de investigación e innovación en
Tecnología Educativa —GITE-USAL_, realiza labores de investigación sobre los ámbitos relacionados con la educación inclusiva, la atención a la diversidad, el estudio de la influencia de las tecnologías de la información y comunicación en los procesos de enseñanza y aprendizaje en diferentes niveles educativos, 
la formación del profesorado, la puesta en práctica de experiencias educativas innovadoras; el análisis y evaluación de las competencias digitales de los estudiantes, y el diseño de

Lady Meléndez-Rodríguez (lady_melendez@ yahoo.com.mx). Catedrática de la Universidad Estatal a Distancia de Costa Rica (UNEDCR) y de la Universidad de Costa Rica (UCR), exasesora nacional de Educación Especial y excoordinadora del Programa de Educación Especial de la UNED-CR. Docente universitaria por más de treinta años. Actualmente es profesora invitada de la Maestría en Educación Inclusiva de la Universidad Casa Grande del Ecuador, como docente investigadora del Programa Latinoamericano de Doctorado en Educación de la UCR y como investigadora del Programa Estado de la Educación, además de coordinar el Observatorio Nacional de la Educación Inclusiva. Consultora y ponente,

Viviana Berrocal-Carvajal (vberrocal@uned. ac.cr). Doctora en Tecnología Instruccional y Educación a Distancia (Nova Southeastern Univerity, 2012). Catedrática de la Universidad Estatal a Distancia, Costa Rica. Actualmente trabaja en la UNED, Costa Rica, como coordi- propuestas de evaluación y desarrollo de dichas competencias. Sus principales ámbitos de docencia son la didáctica general y la atención a la diversidad.

con diversos libros, guías didácticas, manuales y publicaciones en memorias y revistas costarricenses y del exterior, sobre temas relacionados con la teoría de la educación, educación inclusiva, tecnología educativa y neurociencia y educación. Entre sus libros destacan La inclusión escolar del alumno con discapacidad intelectual (2000), Fundamentos y evolución de la educación especial en Costa Rica (2016), Inclusión educativa: Una perspectiva de la didáctica de las Ciencias Naturales (2013). Miembro de la Red RIIE, la Organización Inclusión International, el comité editorial de la revista Actualidades en Psicología de la UCR y el comité editorial de la revista Innovaciones Educativas de su país, entre otros.

nadora de la Maestría en Tecnología Educativa y la carrera de Informática Educativa, donde también realiza labores de investigación sobre el área de la tecnología educativa. Sus principales ámbitos de docencia son la educación a distancia y la tecnología educativa.

\section{Bibliografía}

Aguaded, I.; Marín-Gutiérrez, I. y Caldeiro-Pedreira, M.C. (2018). "Desarrollo de la competencia mediática en el contexto iberoamericano". Revista Letral, 20, pp. 156-182. <http://revistaseug.ugr.es/index.php/letral/ article/view/7814/6847>.

AIMC (2019). Quinto Estudio de la Asociación para la Investigación de Medios de Comunicación, Niñ@s. EGM. <https://bit. ly/2QDALnE>.
Alva de la Rosa, A. (2015). “The New Faces of Inequality in the 21st Century: The Digital Gap". Revista Mexicana de Ciencias Políticas y Sociales, 60(223), pp. 265-285. <https://doi. org/10.1016/S0185-1918(15)72138-0>.

Basco, A. (2017). La tecno-integración de América Latina. Banco Interamericano de Desarrollo (BID). <https://bit.ly/2MQRJxL >.

Binkley, M.; Erstad, O.; Hermna, J.; Raizen, S.; Ripley, M.; Miller-Ricci, M. y Rumble, 
M. (2012). "Defining Twenty-First Century Skills". En: Griffin, P.; Care, E. y McGaw, B. (eds.). Assessment and Teaching of 21st Century Skills. Dordrecht: Springer, pp.17-66.

Bisquerra, R. (coord.) (2004). Metodología de la investigación educativa. Madrid: Editorial La Muralla.

Blau, I. y Shamir-Inbal, T. (2017). "Digital Competences and Long-term ICT Integration in School Culture: The Perspective of Elementary School Leaders". Education and Information Technologies, 22(3), pp. 769-787. <https:// doi.org/10.1007/s10639-015-9456-7>.

Cabero-Almenara, J. y Martínez-Gimeno, A. (2019). "Las TIC y la formación inicial de los docentes. Modelos y competencias digitales". Profesorado, Revista de Currículum y Formación del Profesorado, 23(3), pp. 247-268. <https:// doi.org/10.30827/profesorado.v23i3.9421>.

Carrera, F. J. y Coiduras, J. (2012). "Identificación de la competencia digital del profesor universitario: Un estudio exploratorio en el ámbito de las ciencias sociales". Revista de Docencia Universitaria, 10(2), pp. 273-298. $<$ https://doi.org/10.4995/redu.2012.6108>.

Castañeda, L.; Esteve, F. y Adell, J. (2018). "¿Por qué es necesario repensar la competencia docente para el mundo digital?". Revista de Educación a Distancia, 56, pp. 1-20. <https:// doi.org/10.6018/red/56/6>.

Deursen, A. J. A. M. van y Dijk, J. A. G. M. van (2016). "Modeling Traditional Literacy, Internet Skills and Internet Usage: An Empirical Study". Interacting with Computers, 28(1), pp. 13-26. <https://doi.org/10.1093/ iwc/iwu027>.

Deursen, A. van (2017). "Digital Divide: Impact of Media Literacy". En: VV AA. The International Encyclopedia of Media Effects. Wiley Online Library. <https://doi. org/10.1002/9781118783764.wbieme0044>.

Deursen, A. van; Zeeuw, A. van der; Boer, P.; Jansen, G. y Rompay, T. van (2019). "Digital Inequalities in the Internet of Things: Differences in Attitudes, Material Access, Skills, and Usage". Information, Communication and Socie- ty. <https://doi.org/10.1080/1369118X.2019. 1646777>.

Educational Testing Service (2002). Digital Transformation. A framework for ICT Literacy. A report of the international ICT Literacy Panel. $<$ https://goo.gl/5Qp7eh>.

Esteve, F. y Gisbert, M. (2013). “Competencia digital en la educación superior: Instrumentos de evaluación y nuevos entornos". Enl@ce Revista Venezolana de Información, Tecnología y Conocimiento, 10 (3), pp. 29-43. $<$ https://bit.ly/3aL4V03>.

European Commission (ed.) (2018). Proposal for a Council Recommendation on Key Competences for Lifelong Learning. Oficial Journal of the European Union, C189. <https:// bit.ly/2YsyGNz>.

Fraillon, J.; Schulz, W. y Ainley, J. (2013). International Computer and Information Literacy Study: Assessment Framework. Amsterdam: IEA.

From, J. (2017). “Pedagogical Digital Competence - Between Values, Knowledge and Skills". Higher Education Studies, 7(2), pp. 4350. <https://doi.org/10.5539/hes.v7n2p43>.

García-Ruiz, R. y Pérez-Escoda, A. (2019). "Empoderar a la ciudadanía mediante la educación en medios digitales". Hamut'ay, 6(2), pp. 7-23. <http://dx.doi.org/10.21503/hamu. v6i2.1771>.

Girón-Escudero, V.; Cózar-Gutiérrez, R. y González-Calero, J. A. (2019). “Análisis de la autopercepción sobre el nivel de competencia digital docente en la formación inicial de maestros/as". Revista Electrónica Interuniversitaria de Formación del Profesorado, 22(3), pp. 193-218. <https://doi.org/10.6018/reifop. 373421>.

Güneş, E. y Bahçivan, E. (2018). “A Mixed Research-Based Model for Pre-Service Science Teachers' Digital Literacy: Responses to "Which Beliefs" and "How and Why They Interact" Questions". Computers and Education, 118, pp. 96-106. <https://doi.org/10.1016/j. compedu.2017.11.012>.

Holloway, D.; Green, L. y Livingstone, S. (2013). Zero to Eight: Young Children and Their 
Internet Use. LSE, Londres: EU Kids Online. $<$ https://goo.gl/MNAAPT>.

Hootsuite (2019). The Global State of Digital in 2019. Hootsuite.

Ilomäki, L.; Paavola, S.; Lakkala, M. y Kantosalo, A. (2016). "Digital Competence - An Emergent Boundary Concept for Policy and Educational Research". Education and Information Technologies, 21(3), pp. 655-679. <https:// doi.org/10.1007/s10639-014-9346-4>.

INEE (2018). Panorama de la Educación. Indicadores de la OCDE 2018. Ministerio de Educación y Formación Profesional. <https://bit. ly/2W6LzgH>.

Instefjord, E. y Munthe, E. (2016). "Preparing Pre-Service Teachers to Integrate Technology: An Analysis of the Emphasis on Digital Competence in Teacher Education Curricula". European Journal of Teacher Education, 39(1), pp. 77-93. <https://doi.org/10.1080/0261976 8.2015.1100602>.

INTEF (2017). Marco Común de Competencia Digital Docente Octubre 2017. MECD. $<$ https://bit.ly/2QkURnr>.

ISTE, International Society of Technology in Education (2008). NETS-T, Estándares nacionales de tecnologías de información y comunicación (TIC) para docentes. <https://bit.ly/36mx509>.

Krumsvik, R. J. (2014). "Teacher Educators' Digital Competence". Scandinavian Journal of Educational Research, 58(3), pp. 269-280.

Livingstone, S.; Ólafsson, K.; Helsper, E.; Lupiáñez-Villanueva, F.; Veltri, G. A. y Folkvord, F. (2017). "Maximizing Opportunities and Minimizing Risks for Children Online: The Role of Digital Skills in Emerging Strategies of Parental Mediation". Journal of Communication, 67 (1), pp. 82-105. <https://doi. org/10.1111/jcom.12277>.

Masanet, M. J.; Guerrero-Pico, M. y Establés, M. J. (2019). "From Digital Native to Digital Apprentice. A Case Study of the Transmedia Skills and Informal Learning Strategies of Adolescents in Spain". Learning, Media and Technology. <https://doi.org/10.1080/1743988 4.2019.1641513>.
Muñoz-Carril, P. C.; González-Sanmamed, M. y Fuentes-Abeledo, E. J. (2011). “Competencias tecnológicas del profesorado universitario. Análisis de su formación en ofimática". Educación XXI, 14(2), pp. 157-188. <https:// doi.org/10.5944/educxx1.14.2.249>.

OCDE (2005). La definición y selección de las competencias clave. Resumen ejecutivo. (DeSeCo). <https://bit.ly/2FgudFY>.

OEI (2009). Metas educativas 2021. $<$ https://bit.ly/3bGOWRw>.

Palfrey, J. y Gasser, U. (2010). Born Digital: Understanding the First Generation of Digital $\mathrm{Na}$ tives. Nueva York: Basic Books.

Partnership for 21st Century Skills (2009). P21 Framework Definitions. <https://bit.ly/ 2W87d4g>.

Pérez-Escoda, A.; Aguaded, I. y RodríguezConde, M. J. (2016). "Generación digital vs escuela analógica. Competencias digitales en el currículum de la educación obligatoria". Digital Education Review, 30, pp. 165-183.

Pérez-Escoda, A. y Fernández-Villacencio, N. (2016). "Digital Competence in Use: From DigComp1 to DigComp2". Proceedings of the Fourth International Conference on Technological Ecosystems for Enhancing Multiculturality, pp. 619-624. <https://doi. org/10.1145/3012430.3012583>.

Pérez-Escoda, A.; García-Ruiz, R. y Aguaded, I. (2019). "Dimensions of Digital Literacy Based on Five Models of Development". Cultura y Educación, 31 (2), pp. 232-266. <https://doi.org/10.1080/11356405.2019.16 03274>.

Pettersson, F. (2018). "On the Issues of Digital Competence in Educational Contexts - A Review of Literature". Education and Information Technologies, 23(3), pp. 1.005-1.021. $<$ https://doi.org/10.1007/s10639-017-9649. $3>$.

Programa Estado de la Nación (2017). Sexto Informe del Estado de la Educación. Costa Rica: Programa Estado de la Nación.

PROSIC (2019). Informe Hacia la Sociedad de la Información y el Conocimiento en Costa 
Rica. Universidad de Costa Rica. <https://bit. ly/39AADh6>.

Redecker, C. y Punie, Y. (2017). European Framework for the Digital Competence of Educators: DigCompEdu. Joint Research Centre. <https://bit.ly/2R1tG09>.

Telefónica (2018). Sociedad digital en España 2018. Fundación Telefónica. <https://bit. ly/2rVwu6G>.

Tourón, J.; Martín, D.; Navarro, E.; Pradas, S. e Íñigo, V. (2018). "Validación de constructo de un instrumento para medir la competencia digital docente de los profesores". Re- vista Española de Pedagogía, 76(269), pp. 25-54. <https://doi.org/10.22550/REP76-1-2018-02>.

UNESCO (ed.) (2018). ICT Competency Framework for Teachers. <https://bit. ly/2WD5kLH>.

UNESCO (2019). Living no one Behind. UNESCO. <https://bit.ly/2WVWxYj>.

Valdivieso, T. y Gonzáles, M. A. (2016). “Competencia digital docente: ¿Dónde estamos? Perfil del docente de educación primaria y secundaria. El caso de Ecuador". Píxel-Bit. Revista de Medios y Educación, 49, pp. 57-73, <https:// doi.org/10.12795/pixelbit.2016.i49.04>. 